\title{
Strategic behavior and noncooperative hierarchical control
}

\author{
A.J.T.M. Weeren ${ }^{1, a, *}$, J.M. Schumacher ${ }^{\text {b,c }}$, J.C. Engwerda ${ }^{\mathrm{c}}$ \\ inter for Economic and Social Research, UFSIA, University of Antwerp, Center for Economic and \\ Social Research, Prinsstraat 13, 2000 Antwerp, Belgium \\ ${ }^{\mathrm{b}} \mathrm{CWI}$ (Centre for Mathematics and Computer Science), Amsterdam, Netherlands \\ 'Department of Econometrics, Tilburg University, Tilburg, Netherlands
}

stract

In a dynamic noncooperative two-level hierarchical situation with a single decision .ker on the upper level (a 'coordinator'), the decision makers on the lower level layers') may attempt to achieve an advantage through the effect that their actions have the position of the coordinator. We introduce a model for such 'strategic behavior' It allow us to study the way in which this behavior depends on the choice of a decision $\mathrm{e}$ by the coordinator. The model requires the analysis of an infinite-horizon nonlinear ferential game with a one-dimensional state space. (C) 1999 Elsevier Science B.V. All hts reserved.

L classification: 110

ywords: Hierarchical control; Coordination; Dynamic games

\section{Introduction}

The noncooperative one-leader/several-followers model is relevant for a great umber of social and economic situations, in which a coordinating agent or ntral board is dealing with a number of agents at a lower level who have some

\footnotetext{
*Corresponding author. E-mail: arie.weeren@ufsia.ac.be

${ }^{1}$ The research for this paper was carried out while this author was with the Department of onometrics, Tilburg University, Tilburg, Netherlands.
} 
independent decision power. One interesting aspect of this situation, when considered in a dynamic setting, is that the responsiveness of the leader to the follower's actions may be exploited by the followers for their own purposes. This is what is sometimes called strategic behavior. It is the purpose of this paper to build and analyze a class of models in which such strategic behavior in a noncooperative hierarchical control setting can be studied. It would be too much to expect analytical solutions in this context, even for the relatively simple models that we shall consider, but we shall demonstrate the numerical tractability of the models in the class that we shall describe.

One-leader/several-followers situations occur very frequently in various types of organizations and socio-economic structures, with varying types of division of power between the decision levels. For instance, supranational organizations usually have a central board, which however often serves as a negotiation platform for the member states rather than as a decision unit with independent power. In the case of the European Union, however, it is probably reasonable to say that the European Commission takes to some extent its own decisions. On the national level, the one-leader/several-followers model may for instance be used in a description of the relation between the national government (as leader) and the trade unions and employers (as followers). Here it is clear that each of the involved parties determines its own strategy, with the government in a mediating role whereas the unions and the employers are likely to follow Nash-type strategies with respect to each other. More strictly hierarchical situations can be found for instance in large companies with several business units, but even here one may argue that the business units have their own goals which they will pursue. In other realms of life, one may think of the relation between a referee and the two teams in a sports game; the teams are in some sense subordinate to the referee but are also free to choose the actions they see best fit to achieve victory, whereas the referee does not have such a clear criterion to optimize but does aim for a certain type of stability. In each of the examples given above, the decision makers on the lower level are knowledgeable about the way the leader will respond to their actions, and they will incorporate this knowledge into their decisions. They may even try to 'invest' in a change of attitude of the leader in order to gain in the long run. We shall refer to this type of behavior as strategic behavior.

Situations in which one has one leader and several followers are also studied in hierarchical control theory. In the large body of literature devoted to hierarchical control (see for instance Mesarovic et al. 1970; Jamshidi 1983; Singh 1980), it is typically assumed, however, that the agents at the lower level will simply follow the instructions coming from the upper level. The cooperativity assumption is perfectly valid in many technical systems, but is subject to a Lucas-type critique in the context of social and economic systems. Indeed, the key point in the Lucas (1976) critique of traditional econometric policy evaluation is that the agents in the economy have their own purposes and will plan 
their actions using their knowledge of the expected government's responses. Strategic behavior in the sense used here may be seen as a manifestation of this fact in a deterministic noncooperative hierarchical control setting.

The one-leader/several-followers model is presented in the context of finite games for instance by Başar and Olsder (1995), (Definition 3.35); a study of strategic behavior in our sense however requires a dynamic setting. Such a context is used by Bagchi (1984), (Chapter 5) in a model for a regional investment allocation problem, but he uses an open-loop equilibrium concept which again precludes a study of strategic behavior. Also the 'incentives' model as presented by Ho et al. (1982) does not seem capable of representing strategic behavior, since in this model the objective functions of the followers can be directly altered by the leader. In general the one-leader/several-followers model does not seem to have been used very much in the literature; this may be due to the inherent complexity of the model, which virtually rules out any analytic solutions. In this paper we shall present a numerical solution method, under the admittedly restrictive (but in some circumstances still justifiable) assumption of a one-dimensional state.

In summary, we shall consider a type of problem that from a control theory point of view can be looked at as noncooperative hierarchical control problems, or from a game theory point of view as dynamic games with one leader and several noncooperative followers. To emphasize the nature of the situation, we shall refer to the leader as the 'coordinator' and to the followers as 'players'. We shall work within an infinite-horizon continuous-time setting, and take the stationary feedback Nash equilibrium as a solution concept on the lower level, whereas the strategy of the coordinator will be taken from some parametrized class of strategies. The players are aware of the strategy used by the coordinator, and they will use this knowledge when choosing their own strategies; this is the 'strategic behavior'.

The outline of the paper is as follows. In Section 2 we will develop a general model describing the strategic interactions between players and coordinator. In Section 3 we will discuss solution methods for differential-algebraic equations in general and for HJBI equations in particular. Then in Sections 4 and 5 we present two mechanisms fitting the general model of Section 2. In Section 4 we present a redistribution mechanism in which the coordinator is given direct control over the distribution of the payoffs between the individual players. We will discuss how one can numerically obtain all stationary feedback Nash equilibria of the resulting differential game using the methods developed in Section 3, and illustrate this by a worked example. In Section 5 we present another possible mechanism fitting the general model, which we refer to as the Pareto mechanism. In this case the coordinator influences the choice of Pareto efficient strategy, in such a way that the resulting differential game describes a movement along the Pareto frontier of the underlying static game. Finally, in Section 6 we present some conclusions. 


\section{General model formulation}

\subsection{Introduction}

Consider the following situation. Two players repeatedly play a nonzero-sum game $G$. Assume now that the game $G$ depends in some way (through the payoffs that the players receive, or through the strategy spaces that are available to them) on a parameter $\alpha \in[0,1]$ that may vary in time. The value of $\alpha$ is determined by a 'coordinator' through some decision rule that takes the actions of the players into account. In this way the decisions of the players can influence their future payoffs, and a differential game arises which we shall refer to as the 'controlled game'. Working over an infinite horizon and comparing the asymptotic values of the equilibria of the controlled game to the possible modes of play in the original game $G$, we can see to what extent the decision rule meets the coordinator's objectives.

Example 1. Consider two identical firms producing the same good. The firms realize that it is not efficient to determine the amounts to produce separately. They also know that a kartel-like cooperative like OPEC, in which they both would have to agree on the exact amounts to produce at the given time instant, is not stable, in the sense that there is always an incentive to deviate. So they decide to establish a cooperative in which they both sign an agreement that this cooperative sells the goods on the markets and then shares the payoffs between the firms. The cooperative gives advice to the firms on the amounts to produce. Based on this advice and on the actual amounts produced, the cooperative determines (according to a certain rule that is known to the firms) which share of the profit goes to each firm. Assuming that the cooperative is a price-taker and that the market clears instantaneously, the game $G$ that is played at every time instant is a Cournot duopoly. The parameter $\alpha \in[0,1]$ is the ratio of the profit that goes to firm 1 . The decision rule for the coordinator now involves the choice of $\alpha(t)$ based on the amount of goods produced by both firms at time instant $t$. Global control objectives may be stated for instance in terms of 'fairness' or 'efficiency'.

We formalize this idea as follows. Consider a two-player static game $G$ in strategic form, with strategy spaces $\Gamma_{i}$ and payoff functions $\pi_{i}$. In this game the objective for player $i$ is to maximize his payoff $\pi_{i}$. From this game $G$ we construct a new game, $G(\alpha)$, for every $\alpha$ in $[0,1]$, where $\alpha$ is the variable that is manipulated by the coordinator. Denote by $\Gamma_{i}(\alpha)$ the strategy spaces of $G(\alpha)$ and for each $\alpha \in[0,1]$ by $v_{i}: \Gamma_{1}(\alpha) \times \Gamma_{2}(\alpha) \rightarrow \mathbb{R}$ the payoffs of $G(\alpha)$. Now assume that the coordinator can discriminate between the strategies $\gamma_{i}(\alpha)$ chosen by the individual players, and uses a decision rule $f$ to determine the future values of $\alpha$ based on the observed decisions $\left(\gamma_{1}(\alpha), \gamma_{2}(\alpha)\right)$, 
such that

$$
\dot{\alpha}=f\left(\alpha, \gamma_{1}(\alpha), \gamma_{2}(\alpha)\right) \text {. }
$$

Finally, by choosing as an objective function for the individual players either

$$
\mathscr{L}_{i}=\int_{0}^{t_{s}} v_{i}\left(\alpha(t), \gamma_{1}(\alpha(t)), \gamma_{2}(\alpha(t))\right) \mathrm{d} t
$$

or

$$
\mathscr{L}_{i}=\int_{0}^{\infty} \mathrm{e}^{-r t} v_{i}\left(\alpha(t), \gamma_{1}(\alpha(t)), \gamma_{2}(\alpha(t))\right) \mathrm{d} t
$$

for some $r>0$, a differential game is specified, which we refer to as the controlled game. So the construction of a controlled game from a static game $G$ is done in the following steps:

Step 1: choice of a coordination mechanism $G \mapsto G(\alpha)$,

Step 2: specification of a decision rule $f\left(\alpha, \gamma_{1}, \gamma_{2}\right)$ for the coordinator,

Step 3: choice between a finite-horizon criterion and an infinite-horizon discounted criterion, and in the latter case specification of $r>0$.

\subsection{Construction of a controlled game}

The construction of a controlled game as just described can be carried out in many ways. In this subsection we shall make some more specific choices, which however will still leave open quite a wide range of possibilities. First we make some assumptions on the underlying static game $G$. Note that these assumptions are made for convenience only; by making these assumptions we can reduce the involved technicalities so that we can focus on fundamental issues.

Assumption 2. The strategy spaces $\Gamma_{i} \subseteq \mathbb{R}^{k}$ are convex.

The payoff functions $\pi_{i}: \Gamma_{1} \times \Gamma_{2} \rightarrow \mathbb{R}, i=1,2$, are twice differentiable and strictly concave, i.e.

$$
\left(\begin{array}{cc}
\frac{\partial^{2} \pi_{i}}{\partial \gamma_{1}^{2}} & \frac{\partial^{2} \pi_{i}}{\partial \gamma_{1} \partial \gamma_{2}} \\
\frac{\partial^{2} \pi_{i}}{\partial \gamma_{1} \partial \gamma_{2}} & \frac{\partial^{2} \pi_{i}}{\partial \gamma_{2}^{2}}
\end{array}\right)<0 .
$$

By $\bar{\gamma}=\left(\bar{\gamma}_{1}, \bar{\gamma}_{2}\right) \in \Gamma_{1} \times \Gamma_{2}$ we denote a Nash equilibrium of the game $G$.

For convenience, we assume that the strategy spaces $\Gamma_{i}(\alpha)$ of $G(\alpha)$ are constructed as:

$$
\gamma_{i}(\alpha):=c_{i}(\alpha) \gamma_{i}^{*}(\alpha)+\left(1-c_{i}(\alpha)\right) \gamma_{i}^{\mathrm{a}}(\alpha) .
$$


In doing this, we effectively parametrize the players' decisions for a given $\alpha$ in terms of a variable $c_{i}(\alpha)$ that takes values between 0 and 1 and that can in some applications be interpreted as 'willingness to cooperate'. Denote by $\gamma_{i}^{*}(\alpha)$ a strategy that player $i$ is advised to play in the event the coordinator chooses $\alpha$. Furthermore, denote by $\gamma_{i}^{\mathrm{a}}(\alpha)$ an alternative strategy, that player $i$ would play when playing noncooperatively, i.e. the strategy player $i$ chooses in case he ignores the advice of the coordinator. The issue on how to choose such an alternative strategy is closely related to the issue of choosing threatpoints or disagreement strategies in bargaining theory (see, e.g. van Damme, 1991; Houba, 1994; Osborne and Rubinstein, 1991). A possible choice of alternative strategy is a Nash equilibrium for the underlying game $G$. Especially in the case that $G$ has a unique Nash equilibrium this seems to be a good choice, for the Nash equilibrium is the standard equilibrium concept in noncooperative situations (see, e.g. van Damme, 1991). Another choice might be a best reply against the strategy $\gamma_{j}^{*}(\alpha)$ of the other player.

Intuitively, $c_{i}(\alpha)$ is a parameter reflecting the willingness of player $i$ to follow the advice of the coordinator for given $\alpha$. If $c_{i}(\alpha)=0$ then player $i$ chooses to play the alternative strategy $\gamma_{i}^{\mathrm{a}}(\alpha)$ and if $c_{i}(\alpha)=1$ then player $i$ chooses to play the strategy $\gamma_{i}^{*}(\alpha)$. We allow the players to hesitate between cooperative and noncooperative play by allowing the parameter $c_{i}(\alpha)$ to take values between 0 and 1 . Hence, for given $c_{i}(\alpha)$, the strategy that is actually played by player $i$ is given by Eq. (1).

As before, we assume that the coordinator by observing the actions of both players at time-instant $t$ knows the value of $c_{i}(\alpha(t))$. Using this information the coordinator adjusts the value of $\alpha(t)$. The process of coordination can be described by a decision rule $f$, resulting in the dynamics

$$
\dot{\alpha}(t)=f\left(\alpha(t), \gamma_{1}(\alpha(t)), \gamma_{2}(\alpha(t))\right),
$$

which, by some abuse of notation we will sometimes write as

$$
\dot{\alpha}(t)=f\left(\alpha(t), c_{1}(\alpha(t)), c_{2}(\alpha(t))\right),
$$

thus emphasizing the fact that the choice of $\left(c_{1}(\alpha), c_{2}(\alpha)\right)$ fully determines the pair of strategies $\left(\gamma_{1}(\alpha), \gamma_{2}(\alpha)\right)$.

The decision rule $f$ has to satisfy some properties:

1. $f$ has to be sufficiently smooth, i.e. $f$ has to be at least twice differentiable w.r.t. $c_{i}$, and at least differentiable w.r.t. $\alpha$,

2. $\forall_{c_{1}, c_{2}} f\left(0, c_{1}, c_{2}\right) \geq 0, f\left(1, c_{1}, c_{2}\right) \leq 0$,

3. $\partial^{2} f / \partial c_{i} \partial c_{j}=0, \partial f / \partial c_{i} \not \equiv 0$.

The smoothness condition is imposed in order to prevent some technical difficulties in the sequel of this paper. Clearly this condition might be weakened at the expense of some technical difficulties. The second condition is crucial, in 
the sense that it guarantees that $\alpha(t)$ remains in $[0,1]$ for all $t$. Note that, due to this property, every nontrivial choice for $f$ will be nonlinear. Finally, the third condition is sufficient to guarantee that the optimization problems we will encounter in the sequel of this paper are strictly concave, and that the mechanism is not trivial. Obviously also this condition might be weakened, and in this case a more delicate analysis would be required. An example of a coordination rule satisfying properties $1-3$ is

$$
f\left(\alpha, c_{1}, c_{2}\right)=\beta \alpha(1-\alpha)\left(c_{2}-c_{1}\right),
$$

where $\beta$ is a nonzero constant.

As already remarked in the introduction, an important assumption we make is that both players exactly know the decision rule $f$ the coordinator is using. This opens a possibility for strategic behavior by both players. By choosing $c_{1}$ and $c_{2}$ the players can influence the behavior of the coordinator. A nonlinear differential game emerges, where $\alpha$ is the state variable, $c_{1}$ and $c_{2}$ are the controls, and with the payoff functionals

$$
L_{i}=\int_{0}^{t_{s}} v_{i}\left(\alpha(t), \gamma_{1}(\alpha(t)), \gamma_{2}(\alpha(t))\right) \mathrm{d} t,
$$

in which $\gamma_{i}(\alpha(t))=c_{i}(\alpha(t)) \gamma_{i}^{*}(\alpha(t))+\left(1-c_{i}(\alpha(t))\right) \gamma_{i}^{\mathrm{a}}(\alpha(t))$. This newly defined differential game is the controlled game.

Note that by introducing $\gamma_{i}(\alpha)=c_{i}(\alpha) \gamma_{i}^{*}(\alpha)+\left(1-c_{i}(\alpha)\right) \gamma_{i}^{a}(\alpha)$ the payoff for player $i$ at time instant $t$ is given by $v_{i}\left(\alpha(t), \gamma_{1}(\alpha(t)), \gamma_{2}(\alpha(t))\right)$, which we will sometimes write with some abuse of notation as $v_{i}\left(\alpha(t), c_{1}(t), c_{2}(t)\right)$. In the sequel of this paper we will assume that $v_{1}$ and $v_{2}$ are strictly concave in $\left(c_{1}, c_{2}\right)$. Note again that this concavity assumption is for convenience only; dropping this assumption will only result in a technically more involved analysis.

\subsection{Equilibria of the controlled game}

A natural solution concept to consider for the controlled game is the feedback Nash equilibrium (see Başar and Olsder, 1995). As is well-known (see Başar and Olsder, 1995), feedback Nash equilibria for this differential game correspond to solutions of the Hamilton-Jacobi-Bellman-Isaacs (HJBI) equations

$$
\begin{aligned}
& -\frac{\partial V_{1}}{\partial t}(\alpha, t)=\max _{c_{1} \in[0,1]}\left\{\frac{\partial V_{1}}{\partial \alpha}(\alpha, t) f\left(\alpha, c_{1}, c_{2}\right)+v_{1}\left(\alpha, c_{1}, c_{2}\right)\right\}, \\
& -\frac{\partial V_{2}}{\partial t}(\alpha, t)=\max _{c_{2} \in[0,1]}\left\{\frac{\partial V_{2}}{\partial \alpha}(\alpha, t) f\left(\alpha, c_{1}, c_{2}\right)+v_{2}\left(\alpha, c_{1}, c_{2}\right)\right\},
\end{aligned}
$$

where $V_{1}$ and $V_{2}$ denote value functions. 
Remark 3. We use Eqs. (5) and (6) as a shorthand notation for

$$
\begin{aligned}
& -\frac{\partial V_{1}}{\partial t}(\alpha, t)=\frac{\partial V_{1}}{\partial \alpha}(\alpha, t) f\left(\alpha, c_{1}(\alpha, t), c_{2}(\alpha, t)\right)+v_{1}\left(\alpha, c_{1}(\alpha, t), c_{2}(\alpha, t)\right), \\
& -\frac{\partial V_{2}}{\partial t}(\alpha, t)=\frac{\partial V_{2}}{\partial \alpha}(\alpha, t) f\left(\alpha, c_{1}(\alpha, t), c_{2}(\alpha, t)\right)+v_{2}\left(\alpha, c_{1}(\alpha, t), c_{2}(\alpha, t)\right)
\end{aligned}
$$

in which

$$
\begin{aligned}
& c_{1}(\alpha, t) \in \underset{c_{1} \in[0,1]}{\arg \max }\left\{\frac{\partial V_{1}}{\partial \alpha}(\alpha, t) f\left(\alpha, c_{1}, c_{2}(\alpha, t)\right)+v_{1}\left(\alpha, c_{1}, c_{2}(\alpha, t)\right)\right\}, \\
& c_{2}(\alpha, t) \in \underset{c_{2} \in[0,1]}{\arg \max }\left\{\frac{\partial V_{2}}{\partial \alpha}(\alpha, t) f\left(\alpha, c_{1}(\alpha, t), c_{2}\right)+v_{2}\left(\alpha, c_{1}(\alpha, t), c_{2}\right)\right\} .
\end{aligned}
$$

The same shorthand notation will be used in similar cases below.

In order to facilitate our analysis, we will consider the controlled game over an infinite time horizon, with discounted payoffs. This produces the payoff functionals

$$
L_{i}=\int_{0}^{\infty} \mathrm{e}^{-r t} v_{i}\left(\alpha(t), \gamma_{1}(\alpha(t)), \gamma_{2}(\alpha(t))\right) \mathrm{d} t,
$$

where $\gamma_{i}(\alpha(t))=c_{i}(\alpha(t)) \gamma_{i}^{*}(\alpha(t))+\left(1-c_{i}(\alpha(t))\right) \gamma_{i}^{a}(\alpha(t))$. Moreover we shall restrict our attention to stationary feedback Nash equilibria ${ }^{2}$ corresponding to continuously differentiable value functions.

Now the HJBI equations describing stationary feedback Nash equilibria (see, e.g. Feichtinger and Wirl, 1993; Tsutsui and Mino, 1990) reduce to the system

$$
\begin{aligned}
& r V_{1}(\alpha)=\max _{c_{1} \in[0,1]}\left\{V_{1}^{\prime}(\alpha) f\left(\alpha, c_{1}, c_{2}\right)+v_{1}\left(\alpha, c_{1}, c_{2}\right)\right\}, \\
& r V_{2}(\alpha)=\max _{c_{2} \in[0,1]}\left\{V_{2}^{\prime}(\alpha) f\left(\alpha, c_{1}, c_{2}\right)+v_{2}\left(\alpha, c_{1}, c_{2}\right)\right\},
\end{aligned}
$$

where of course also remark 3 applies.

Remark 4. The characterization of stationary feedback Nash equilibria by the HJBI Eqs. (12) and (13) must be understood in the following way. It can be shown that if $\left(\bar{V}_{1}, \bar{V}_{2}, \bar{c}_{1}, \bar{c}_{2}\right)$ are continuously differentiable solutions of Eqs. (12)

\footnotetext{
${ }^{2}$ In Feichtinger and Wirl, (1993), Maskin and Tirole (1994), Tsutsui and Mino, (1990), these are called Markov perfect Nash equilibria.
} 
and (13) such that $\bar{V}_{1}$ and $\bar{V}_{2}$ are bounded, then the pair of strategies $\left(\bar{c}_{1}, \bar{c}_{2}\right)$ is a stationary feedback Nash equilibrium (see also Tsutsui and Mino, 1990).

Remark 5 . Note that by requiring stationary feedback Nash equilibria, Folktheorem-like results do not immediately apply, for trigger strategies are not admissible (see Maskin and Tirole (1994)). Nevertheless, stationary feedback Nash equilibria are in general not unique (see Feichtinger and Wirl, 1993; Tsutsui and Mino, 1990; Weeren et al., 1994).

By the assumption that $v_{1}$ and $v_{2}$ are strictly concave in $c_{1}$ and $c_{2}$ and by the assumptions on $f$, system (12), (13) is equivalent to the system

$$
\begin{aligned}
& \frac{\partial f}{\partial c_{1}}\left(\alpha, c_{1}, c_{2}\right) V_{1}^{\prime}(\alpha)+\frac{\partial v_{1}}{\partial c_{1}}\left(\alpha, c_{1}, c_{2}\right)=\eta_{1}, \\
& \frac{\partial f}{\partial c_{2}}\left(\alpha, c_{1}, c_{2}\right) V_{2}^{\prime}(\alpha)+\frac{\partial v_{2}}{\partial c_{2}}\left(\alpha, c_{1}, c_{2}\right)=\eta_{2}, \\
& f\left(\alpha, c_{1}, c_{2}\right) V_{1}^{\prime}(\alpha)+v_{1}\left(\alpha, c_{1}, c_{2}\right)-r V_{1}(\alpha)=0, \\
& f\left(\alpha, c_{1}, c_{2}\right) V_{2}^{\prime}(\alpha)+v_{2}\left(\alpha, c_{1}, c_{2}\right)-r V_{2}(\alpha)=0, \\
& 0 \leq c_{1} \leq 1,\left(1-c_{1}\right) \eta_{1} \leq 0, c_{1} \eta_{1} \geq 0, \\
& 0 \leq c_{2} \leq 1,\left(1-c_{2}\right) \eta_{2} \leq 0, c_{2} \eta_{2} \geq 0 .
\end{aligned}
$$

In case no constraint on $c_{1}$ and $c_{2}$ is active, this results in the system of differential-algebraic equations

$$
\begin{aligned}
& \frac{\partial f}{\partial c_{1}}\left(\alpha, c_{1}, c_{2}\right) V_{1}^{\prime}(\alpha)+\frac{\partial v_{1}}{\partial c_{1}}\left(\alpha, c_{1}, c_{2}\right)=0, \\
& \frac{\partial f}{\partial c_{2}}\left(\alpha, c_{1}, c_{2}\right) V_{2}^{\prime}(\alpha)+\frac{\partial v_{2}}{\partial c_{2}}\left(\alpha, c_{1}, c_{2}\right)=0, \\
& f\left(\alpha, c_{1}, c_{2}\right) V_{1}^{\prime}(\alpha)+v_{1}\left(\alpha, c_{1}, c_{2}\right)-r V_{1}(\alpha)=0, \\
& f\left(\alpha, c_{1}, c_{2}\right) V_{2}^{\prime}(\alpha)+v_{2}\left(\alpha, c_{1}, c_{2}\right)-r V_{2}(\alpha)=0 .
\end{aligned}
$$

The equations above constitute four differential/algebraic equations in four unknowns, to wit $V_{1}(\alpha), V_{2}(\alpha), c_{1}(\alpha)$, and $c_{2}(\alpha)$. Because the equations are DAEs rather than ODEs, the number of required initial conditions is not four but rather two in most cases, as will be shown below. The equations will in general have a unique solution for a given initial condition; the freedom in choosing the initial condition corresponds to the nonuniqueness of feedback Nash equilibria (cf. van Damme, 1991). Once a solution is obtained, the functional dependence of $c_{1}$ and $c_{2}$ on $\alpha$ is established and the evolution of $\alpha$ as a function of $t$ can be 
obtained from Eq. (3), in which $c_{1}$ and $c_{2}$ depend on $t$ through $\alpha$. Subsequently, the evolution in time of $c_{1}$ and $c_{2}$ is obtained, and in particular one finds the limit values of $c_{1}$ and $c_{2}$ as $t$ tends to infinity. It is then of interest, for instance, to study the dependence of these limit values on the choice of a Nash equilibrium and on the strategy of the coordinator. The process will be illustrated in several examples below.

\section{Analysis of HJBI-DAEs}

In the current section we will discuss the (numerical) treatment of the $\mathrm{HJBI}$ DAEs. For a extensive treatment of general DAEs the interested reader is referred to Brasey and Hairer (1993), Brenan et al. (1989), Gear (1988), Griepentrog and März (1986), Hairer et al. (1989) and Hairer and Wanner (1991).

By a differential-algebraic equation (DAE) is meant an equation of the form

$$
F\left(t, y, y^{\prime}\right)=0
$$

in which $y$ is a function of $t$, and $y^{\prime}$ is the first derivative of $y$ with respect to $t$. First we take a look at the system of HJBI-DAEs, Eqs. (18)-(21), i.e. the system of HJBI equations in case the constraints $c_{1} \geq 0, c_{1} \leq 1, c_{2} \geq 0$ and $c_{2} \leq 1$ are not active. For ease of notation, we will suppress the arguments of $f, V_{i}$, and $v_{i}$ in the rest of this section. Define

$$
y:=\left(\begin{array}{l}
V_{1} \\
V_{2} \\
c_{1} \\
c_{2}
\end{array}\right) .
$$

We first compute the Jacobian $F_{y^{\prime}}$,

$$
F_{y^{\prime}}=\left(\begin{array}{cccc}
\frac{\partial f}{\partial c_{1}} & 0 & 0 & 0 \\
0 & \frac{\partial f}{\partial c_{2}} & 0 & 0 \\
f & 0 & 0 & 0 \\
0 & f & 0 & 0
\end{array}\right)
$$

Clearly $F_{y^{\prime}}$ is not invertible, so the system (18)-(21) is not an (implicit) ODE. Differentiating the system of HJBI-DAEs once, using $\partial^{2} f / \partial c_{i} \partial c_{j}=0$ and Eqs. (18) and (19), we obtain the additional equations

$$
\frac{\partial^{2} f}{\partial \alpha \partial c_{1}} V_{1}^{\prime}+\frac{\partial f}{\partial c_{1}} V_{1}^{\prime \prime}+\frac{\partial^{2} v_{1}}{\partial \alpha \partial c_{1}}+\frac{\partial^{2} v_{1}}{\partial c_{1}^{2}} c_{1}^{\prime}+\frac{\partial^{2} v_{1}}{\partial c_{1} \partial c_{2}} c_{2}^{\prime}=0
$$




$$
\begin{aligned}
& \frac{\partial^{2} f}{\partial \alpha \partial c_{2}} V_{2}^{\prime}+\frac{\partial f}{\partial c_{2}} V_{2}^{\prime \prime}+\frac{\partial^{2} v_{2}}{\partial \alpha \partial c_{2}}+\frac{\partial^{2} v_{2}}{\partial c_{1} \partial c_{2}} c_{1}^{\prime}+\frac{\partial^{2} v_{2}}{\partial c_{2}^{2}} c_{2}^{\prime}=0, \\
& f V_{1}^{\prime \prime}-\left(\frac{\partial f}{\partial \alpha}-r\right)\left(\frac{\partial v_{1}}{\partial c_{1}} / \frac{\partial f}{\partial c_{1}}\right)+\frac{\partial v_{1}}{\partial \alpha}+\left(\frac{\partial v_{1}}{\partial c_{2}}-\frac{\partial f}{\partial c_{2}} \frac{\partial v_{1}}{\partial c_{1}} / \frac{\partial f}{\partial c_{1}}\right) c_{2}^{\prime}=0, \\
& f V_{2}^{\prime \prime}-\left(\frac{\partial f}{\partial \alpha}-r\right)\left(\frac{\partial v_{2}}{\partial c_{2}} / \frac{\partial f}{\partial c_{2}}\right)+\frac{\partial v_{2}}{\partial \alpha}+\left(\frac{\partial v_{2}}{\partial c_{1}}-\frac{\partial f}{\partial c_{1}} \frac{\partial v_{2}}{\partial c_{2}} / \frac{\partial f}{\partial c_{2}}\right) c_{1}^{\prime}=0 .
\end{aligned}
$$

Note that we can eliminate $V_{1}^{\prime \prime}$ and $V_{2}^{\prime \prime}$ from Eqs. (27) and (28) using Eqs. (25) and (26). Using this elimination we can straightforwardly derive that Eqs. (27) and (28) constitute an implicit ODE for $c_{1}^{\prime}$ and $c_{2}^{\prime}$ if and only if the matrix

$$
\mathscr{J}:=\left(\begin{array}{cc}
-f \frac{\partial^{2} v_{1}}{\partial c_{1}^{2}} / \frac{\partial f}{\partial c_{1}} & \frac{\partial v_{1}}{\partial c_{2}}-\frac{\partial}{\partial c_{2}}\left(f \frac{\partial v_{1}}{\partial c_{1}}\right) / \frac{\partial f}{\partial c_{1}} \\
\frac{\partial v_{2}}{\partial c_{1}}-\frac{\partial}{\partial c_{1}}\left(f \frac{\partial v_{2}}{\partial c_{2}}\right) / \frac{\partial f}{\partial c_{2}} & -f \frac{\partial^{2} v_{2}}{\partial c_{2}^{2}} / \frac{\partial f}{\partial c_{2}}
\end{array}\right)
$$

is nonsingular. The procedure we have followed for the system (18)-(21), can in general be applied to any DAE. Related to this procedure the so-called (differential) index is introduced: the index of a DAE is, loosely speaking, the (minimal) number of times the system (22) has to be differentiated in order to obtain a (possibly implicit) ODE. For a more formal treatment of the index see, e.g. Griepentrog and März (1986) and Brenan et al. (1989). The index is of crucial importance in selecting a numerical solution method for a given DAE. Backward differentiation formulas (BDF) have emerged as the most popular and best understood class of linear multistep methods for DAEs (see Brenan et al., 1989; Hairer and Wanner, 1991). In general, multistep methods and Runge-Kutta methods are not stable for higher-index DAE systems. In the case of a system of DAEs of index 0 or 1 it is always possible to use these methods. A well-known implementation of the BDF technique is provided in the Fortran package DASSL (as described in Brenan et al. (1989)). For the treatment of higher-index systems the reader is referred to Brasey and Hairer (1993), Brenan et al. (1989), Gear (1988), Hairer et al. (1989) and Hairer and Wanner (1991).

Remark 6. Note that the method of studying HJBI equations via the so-called auxiliary equations as introduced in Tsutsui and Mino, (1990) is closely related to the setup described in this section. The model considered in Tsutsui and Mino, (1990) is of a more special form than the one considered here, which makes it possible to obtain explicit expressions for the equilibrium feedback strategies and to substitute these in the HJBI equations. Then, by differentiating the HJBI equations (implicit) ODEs are obtained. These ODEs have the 
property that they do not depend on $V_{1}$ and $V_{2}$. After deriving the ODEs, symmetry conditions are used to reduce the system of ODEs to a single first-order ODE in $y=V_{1}^{\prime}=V_{2}^{\prime}$. This ODE is then solved analytically. In Feichtinger and Wirl, (1993) a similar setup is used.

Recapitulating we now have the following result for the system (18)-(21).

Proposition 7. The system of HJBI-DAEs, Eqs. (18)-(21), has index 1 if and only if the matrix $\mathscr{I}$ given by Eq. (29) is nonsingular.

The systems of DAEs which emerge when one of the constraints $c_{1} \geq 0$, $c_{1} \leq 1, c_{2} \geq 0$ or $c_{2} \leq 1$ becomes active, can be shown to have index at least one in a similar fashion. Moreover, conditions as described in Proposition 7 can be derived.

In case two constraints are active, the equations are either index 0 (i.e. implicit ODEs) or algebraic, depending on whether $f\left(\alpha, c_{1}, c_{2}\right)=0$ or not.

\section{A redistribution mechanism}

As already discussed in Section 2, there are several ways in which the coordination parameter $\alpha$ may affect the underlying static game $G$. In this section we consider the case in which the payoffs depend on $\alpha$, but $\gamma^{*}$ and $\gamma^{\mathrm{a}}$ do not. Moreover, we will concentrate on the control objective of cooperative behavior. This objective can be chosen in order to maximize efficiency. In this section we will show how for this control objective a controlled game can be specified and analyzed. Special attention is payed to the HJBI-DAEs that arise for this case. For a particular choice of game $G$ and decision rule $f$ we illustrate the numerical method.

\subsection{A symmetric redistribution game}

We make the following assumptions about the underlying static game $G$.

Assumption 8. (i) The game $G$ is symmetric, i.e. $\Gamma_{1}=\Gamma_{2}$ and $\pi_{1}\left(\gamma_{1}, \gamma_{2}\right)=$ $\pi_{2}\left(\gamma_{2}, \gamma_{1}\right)$

(ii) $G$ has a unique Nash equilibrium given by $\left(\bar{\gamma}_{1}, \bar{\gamma}_{2}\right)$, with equilibrium payoffs $\left(\bar{\pi}_{1}, \bar{\pi}_{2}\right)$,

(iii) the unique Nash equilibrium of $G$ is not Pareto efficient.

The symmetry suggests restricting our attention to Pareto efficient strategies $\hat{\gamma}\left(\frac{1}{2}\right)$ corresponding to $\mu=\frac{1}{2}$ (see Theorem 13). So for the cooperative strategy we 
choose $\gamma_{i}^{*}(\alpha)=\hat{\gamma}_{i}\left(\frac{1}{2}\right)$. The second assumption, that $G$ has a unique Nash equilibrium, justifies the choice of this Nash equilibrium as the alternative strategy, i.e. $\gamma_{i}^{\mathrm{a}}(\alpha)=\bar{\gamma}_{i}$. Note that both the cooperative strategies $\gamma^{*}$ and the alternative strategies $\gamma^{\mathrm{a}}$ do not depend on $\alpha$ in this case. The extra payoffs from playing $\gamma_{i}(\alpha)=c_{i}(\alpha) \hat{\gamma}_{i}\left(\frac{1}{2}\right)+\left(1-c_{i}(\alpha)\right) \bar{\gamma}_{i}$ are given by

$$
\pi^{*}\left(c_{1}, c_{2}\right):=\pi_{1}\left(\gamma_{1}(\alpha), \gamma_{2}(\alpha)\right)+\pi_{2}\left(\gamma_{1}(\alpha), \gamma_{2}(\alpha)\right)-\pi_{1}\left(\bar{\gamma}_{1}, \bar{\gamma}_{2}\right)-\pi_{2}\left(\bar{\gamma}_{1}, \bar{\gamma}_{2}\right) .
$$

Now suppose that these extra payoffs are redistributed over the players by the coordinator, according to the rule

$$
\begin{aligned}
& v_{1}\left(\alpha, c_{1}, c_{2}\right):=\alpha \pi^{*}\left(c_{1}, c_{2}\right), \\
& v_{2}\left(\alpha, c_{1}, c_{2}\right):=(1-\alpha) \pi^{*}\left(c_{1}, c_{2}\right) .
\end{aligned}
$$

Then the HJBI equations describing the stationary feedback Nash equilibria of the controlled game are given by

$$
\begin{aligned}
& r V_{1}(\alpha)=\max _{c_{1} \in[0,1]}\left\{V_{1}^{\prime}(\alpha) f\left(\alpha, c_{1}, c_{2}\right)+\alpha \pi^{*}\left(c_{1}, c_{2}\right)\right\}, \\
& r V_{2}(\alpha)=\max _{c_{2} \in[0,1]}\left\{V_{2}^{\prime}(\alpha) f\left(\alpha, c_{1}, c_{2}\right)+(1-\alpha) \pi^{*}\left(c_{1}, c_{2}\right)\right\},
\end{aligned}
$$

or, as long as the constraints $c_{1} \geq 0, c_{1} \leq 1, c_{2} \geq 0, c_{2} \leq 1$ are not active, in the form (18)-(21):

$$
\begin{aligned}
& \frac{\partial f}{\partial c_{1}} V_{1}^{\prime}+\alpha \frac{\partial \pi^{*}}{\partial c_{1}}=0, \\
& \frac{\partial f}{\partial c_{2}} V_{2}^{\prime}+(1-\alpha) \frac{\partial \pi^{*}}{\partial c_{2}}=0, \\
& f V_{1}^{\prime}+\alpha \pi^{*}-r V_{1}=0, \\
& f V_{2}^{\prime}+(1-\alpha) \pi^{*}-r V_{2}=0 .
\end{aligned}
$$

As the coordinator's decision rule, we shall take

$$
f\left(\alpha, c_{1}, c_{2}\right)=\beta \alpha(1-\alpha)\left(c_{2}-c_{1}\right),
$$

with $\beta \neq 0$. Now, if we solve Eqs. (35) and (36) for $\left(V_{1}^{\prime}, V_{2}^{\prime}\right)$, and then substitute the result in Eqs. (37) and (38), we obtain

$$
\begin{aligned}
& -\beta \alpha(1-\alpha) V_{1}^{\prime}+\alpha \frac{\partial \pi^{*}}{\partial c_{1}}=0 \\
& \beta \alpha(1-\alpha) V_{2}^{\prime}+(1-\alpha) \frac{\partial \pi^{*}}{\partial c_{2}}=0
\end{aligned}
$$




$$
\begin{aligned}
& \alpha\left(c_{2}-c_{1}\right) \frac{\partial \pi^{*}}{\partial c_{1}}+\alpha \pi^{*}-r V_{1}=0, \\
& -(1-\alpha)\left(c_{2}-c_{1}\right) \frac{\partial \pi^{*}}{\partial c_{2}}+(1-\alpha) \pi^{*}-r V_{2}=0 .
\end{aligned}
$$

The matrix $\mathscr{J}$ (see Eq. (29)) is given by

$$
\mathscr{J}:=\left(\begin{array}{cc}
\alpha\left(c_{2}-c_{1}\right) \frac{\partial^{2} \pi^{*}}{\partial c_{1}^{2}} & \alpha\left(\frac{\partial \pi^{*}}{c_{1}}+\frac{\partial \pi^{*}}{\partial c_{2}}+\left(c_{2}-c_{1}\right) \frac{\partial^{2} \pi^{*}}{\partial c_{1} \partial c_{2}}\right) \\
(1-\alpha)\left(\frac{\partial \pi^{*}}{c_{1}}+\frac{\partial \pi^{*}}{\partial c_{2}}-\left(c_{2}-c_{1}\right) \frac{\partial^{2} \pi^{*}}{\partial c_{1} \partial c_{2}}\right) & -(1-\alpha)\left(c_{2}-c_{1}\right) \frac{\partial^{2} \pi^{*}}{\partial c_{2}^{2}}
\end{array}\right)
$$

Note that $\mathscr{J}$ is nonsingular for all $\alpha \in(0,1)$ if and only if the matrix $\tilde{\mathscr{J}}$ given by

$$
\tilde{J}:=\left(\begin{array}{cc}
0 & \frac{\partial \pi^{*}}{\partial c_{1}}+\frac{\partial \pi^{*}}{\partial c_{2}} \\
-\left(\frac{\partial \pi^{*}}{\partial c_{1}}+\frac{\partial \pi^{*}}{\partial c_{2}}\right) & 0
\end{array}\right)+\left(c_{2}-c_{1}\right)\left(\begin{array}{cc}
\frac{\partial^{2} \pi^{*}}{\partial c_{1}^{2}} & \frac{\partial^{2} \pi^{*}}{\partial c_{1} \partial c_{2}} \\
\frac{\partial^{2} \pi^{*}}{\partial c_{1} \partial c_{2}} & \frac{\partial^{2} \pi^{*}}{\partial c_{2}^{2}}
\end{array}\right)
$$

is nonsingular. Before we show that the system of HJBI-DAEs, Eqs. (39)-(42), is an index 1 system, we first need the following lemma:

Lemma 9. The function $\pi^{*}$ defined in Eq. (30) is strictly concave, i.e. the matrix

$$
\left(\begin{array}{ll}
\frac{\partial^{2} \pi^{*}}{\partial c_{1}^{2}} & \frac{\partial^{2} \pi^{*}}{\partial c_{1} \partial c_{2}} \\
\frac{\partial^{2} \pi^{*}}{\partial c_{1} \partial c_{2}} & \frac{\partial^{2} \pi^{*}}{\partial c_{2}^{2}}
\end{array}\right)
$$

is negative definite.

Proof. The symmetry of the game $G$ implies $\bar{\gamma}_{1}=\bar{\gamma}_{2}=: \bar{\gamma}$ and $\hat{\gamma}_{1}\left(\frac{1}{2}\right)=\hat{\gamma}_{2}\left(\frac{1}{2}\right)=: \hat{\gamma}$. Then elementary calculus shows that

$$
\left(\begin{array}{cc}
\frac{\partial^{2} \pi^{*}}{\partial c_{1}^{2}} & \frac{\partial^{2} \pi^{*}}{\partial c_{1} \partial c_{2}} \\
\frac{\partial^{2} \pi^{*}}{\partial c_{1} \partial c_{2}} & \frac{\partial^{2} \pi^{*}}{\partial c_{2}^{2}}
\end{array}\right)=(\hat{\gamma}-\bar{\gamma})^{2}\left(\left(\begin{array}{cc}
\frac{\partial^{2} \pi_{1}}{\partial \gamma_{1}^{2}} & \frac{\partial^{2} \pi_{1}}{\partial \gamma_{1} \partial \gamma_{2}} \\
\frac{\partial^{2} \pi_{1}}{\partial \gamma_{1} \partial \gamma_{2}} & \frac{\partial^{2} \pi_{1}}{\partial \gamma_{2}^{2}}
\end{array}\right)+\left(\begin{array}{cc}
\frac{\partial^{2} \pi_{2}}{\partial \gamma_{1}^{2}} & \frac{\partial^{2} \pi_{2}}{\partial \gamma_{1} \partial \gamma_{2}} \\
\frac{\partial^{2} \pi_{2}}{\partial \gamma_{1} \partial \gamma_{2}} & \frac{\partial^{2} \pi_{2}}{\partial \gamma_{2}^{2}}
\end{array}\right)\right)
$$


From the strict concavity of $\pi_{1}$ and $\pi_{2}$ it follows that

$$
\left(\begin{array}{cc}
\frac{\partial^{2} \pi_{i}}{\partial \gamma_{1}^{2}} & \frac{\partial^{2} \pi_{i}}{\partial \gamma_{1} \partial \gamma_{2}} \\
\frac{\partial^{2} \pi_{i}}{\partial \gamma_{1} \partial \gamma_{2}} & \frac{\partial^{2} \pi_{i}}{\partial \gamma_{2}^{2}}
\end{array}\right)<0,
$$

for $i=1,2$, and hence

$$
\left(\begin{array}{ll}
\frac{\partial^{2} \pi^{*}}{\partial c_{1}^{2}} & \frac{\partial^{2} \pi^{*}}{\partial c_{1} \partial c_{2}} \\
\frac{\partial^{2} \pi^{*}}{\partial c_{1} \partial c_{2}} & \frac{\partial^{2} \pi^{*}}{\partial c_{2}^{2}}
\end{array}\right)<0
$$

since $\hat{\gamma} \neq \bar{\gamma}$ by Assumption 8(iii).

Proposition 10. The system of HJBI-DAEs, Eqs. (39)-(42), has index 1 on its domain of validity $(0,1) \times(0,1)$.

Proof. We will show that the matrix $\tilde{\mathscr{J}}$ appearing in Eq. (43) is nonsingular for all $\alpha \in(0,1)$. We will consider two cases, first the case $c_{1} \neq c_{2}$, and secondly the case $c_{1}=c_{2}$. In the case $c_{1} \neq c_{2}$ we note that $\tilde{J}$ is the sum of a skew-symmetric matrix and a matrix that is, depending on the sign of $c_{2}-c_{1}$, either positive or negative definite. It follows that the quadratic form determined by $\tilde{J}$ is sign definite, and in particular this implies that, for $c_{1} \neq c_{2}, \tilde{J}$ is nonsingular. In the case $c_{1}=c_{2}$, we see that

$$
\tilde{\mathscr{J}}=\left(\begin{array}{cc}
0 & \frac{\partial \pi^{*}}{\partial c_{1}}+\frac{\partial \pi^{*}}{\partial c_{2}} \\
-\left(\frac{\partial \pi^{*}}{\partial c_{1}}+\frac{\partial \pi^{*}}{\partial c_{2}}\right) & 0
\end{array}\right) .
$$

Note that $\pi^{*}\left(c_{1}, c_{2}\right)=\pi^{*}\left(c_{2}, c_{1}\right)$, and hence $\tilde{y}$ is singular if and only if $\partial \pi^{*} / \partial c_{1}=\partial \pi^{*} / \partial c_{2}=0$. Elementary calculus shows that $\partial \pi^{*} / \partial c_{1}=\partial \pi^{*} / \partial c_{2}=0$ if and only if

$$
\begin{aligned}
& \frac{\partial \pi_{1}}{\partial \gamma_{1}}\left((\alpha), \gamma_{2}(\alpha)\right)+\frac{\partial \pi_{2}}{\partial \gamma_{1}}\left((\alpha), \gamma_{2}(\alpha)\right)=0, \\
& \frac{\partial \pi_{1}}{\partial \gamma_{2}}\left(\gamma_{1}(\alpha), \gamma_{2}(\alpha)\right)+\frac{\partial \pi_{2}}{\partial \gamma_{2}}\left(\gamma_{1}(\alpha), \gamma_{2}(\alpha)\right)=0 .
\end{aligned}
$$


Note that these last equations are (see Theorem 13) precisely the first-order conditions characterizing $\hat{\gamma}\left(\frac{1}{2}\right)$, and hence satisfied if and only if $c_{1}=c_{2}=1$. However, $c_{1}=c_{2}=1$ lies outside the domain of validity $(0,1) \times(0,1)$.

Because the system of HJBI-DAEs, Eqs. (39)-(42), has index 1, we can derive ODEs for $c_{1}$ and $c_{2}$ by differentiating Eqs. (39)-(42) once. The resulting ODEs are given by

$$
\left(\begin{array}{l}
c_{1}^{\prime} \\
c_{2}^{\prime}
\end{array}\right)=\mathscr{J}^{-1}\left(\begin{array}{l}
\left(-\left(c_{2}-c_{1}\right)-\frac{r}{\beta(1-\alpha)}\right) \frac{\partial \pi^{*}}{\partial c_{1}}+\pi^{*} \\
\left(\left(c_{2}-c_{1}\right)-\frac{r}{\beta \alpha}\right) \frac{\partial \pi^{*}}{\partial c_{2}}+\pi^{*}
\end{array}\right)
$$

We shall be interested in particular in symmetric solutions, i.e. those for which $c_{1}(\alpha)=c_{2}(1-\alpha)$ and $V_{1}(\alpha)=V_{2}(1-\alpha)$. These solutions can be characterized as follows.

Lemma 11. A solution $\left(V_{1}, V_{2}, c_{1}, c_{2}\right)$ of the HJBI-DAES, Eqs. (39)-(42), is symmetric if and only if it satisfies $c_{1}\left(\frac{1}{2}\right)=c_{2}\left(\frac{1}{2}\right)$.

Proof. By writing the HJBI-DAEs, Eqs. (39)-(42), and its first derivatives, evaluated in $\alpha=\frac{1}{2}$, it is easily verified that there are only 2 degrees of freedom in specifying consistent initial ${ }^{3}$ conditions, i.e. when 2 variables out of

$$
\left\{V_{1}\left(\frac{1}{2}\right), V_{2}\left(\frac{1}{2}\right), c_{1}\left(\frac{1}{2}\right), c_{2}\left(\frac{1}{2}\right), V_{1}^{\prime}\left(\frac{1}{2}\right), V_{2}^{\prime}\left(\frac{1}{2}\right), c_{1}^{\prime}\left(\frac{1}{2}\right), c_{2}^{\prime}\left(\frac{1}{2}\right)\right\}
$$

are chosen, the other variables are fixed by the system of HJBI-DAEs, Eqs. (39)-(42), and its first derivatives, evaluated in $\alpha=1 / 2$.

Now let $\left(V_{1}(\alpha), V_{2}(\alpha), c_{1}(\alpha), c_{2}(\alpha)\right)$ be a solution of the HJBI-DAEs. Eqs. (39)-(42), corresponding to the initial conditions $\left(c_{1}\left(\frac{1}{2}\right), c_{2}\left(\frac{1}{2}\right)\right)$. Then it can straightforwardly be shown that $\left(V_{2}(1-\alpha), V_{1}(1-\alpha), c_{2}(1-\alpha), c_{1}(1-\alpha)\right)$ is a solution of the HJBI-DAEs, Eqs. (39)-(42), corresponding to the initial conditions $\left(c_{2}\left(\frac{1}{2}\right), c_{1}\left(\frac{1}{2}\right)\right)$. From Eq. (44) we see, because

$$
g\left(\alpha, c_{1}, c_{2}\right):=\mathscr{J}^{-1}\left(\begin{array}{c}
\left.-\left(c_{2}-c_{1}\right)-\frac{r}{\beta(1-\alpha)}\right) \frac{\partial \pi^{*}}{\partial c_{1}}+\pi^{*} \\
\left(\left(c_{2}-c_{1}\right)-\frac{r}{\beta \alpha}\right) \frac{\partial \pi^{*}}{\partial c_{2}}+\pi^{*}
\end{array}\right)
$$

\footnotetext{
${ }^{3}$ Note that in this case we consider $\alpha=1 / 2$ as the 'starting point', i.e. initial conditions are specified in $\alpha=1 / 2$.
} 
is a $C^{1}$ function and hence satisfies a Lipschitz condition, that whenever $c_{1}\left(\frac{1}{2}\right)=c_{2}\left(\frac{1}{2}\right)$, necessarily $c_{1}(\alpha)=c_{2}(1-\alpha)$ for all $\alpha \in(0,1)$.

\subsection{A worked example}

In this subsection we will construct a redistribution controlled game describing a situation as sketched in Example 1. Hence we choose for $G$ a Cournot duopoly. To be specific, let us assume that the price equation is

$$
p(y)= \begin{cases}120-y & \text { if } y \leq 120, \\ 0 & \text { if } y>120\end{cases}
$$

and that the production costs of the individual firms are given by

$$
c_{i}\left(y_{i}\right)=y_{i}^{2} .
$$

Then the payoffs of $G$ are given by (see, e.g. Gibbons 1992; Takayama 1985):

$$
\pi_{i}\left(y_{1}, y_{2}\right)=y_{i}\left(120-y_{1}-y_{2}\right)-y_{i}^{2} .
$$

and consequently the Nash equilibrium $\bar{\gamma}$ of $G$, with payoff $\bar{\pi}$, and the Pareto efficient strategy $\hat{\gamma}\left(\frac{1}{2}\right)$, with payoff $\hat{\pi}$, are given by

$$
\begin{aligned}
& \bar{\gamma}=24, \\
& \bar{\pi}=1152, \\
& \hat{\gamma}\left(\frac{1}{2}\right)=20, \\
& \hat{\pi}=1200 .
\end{aligned}
$$

Hence, the additional payoffs after redistribution (30) are

$$
\pi^{*}\left(c_{1}, c_{2}\right)=96\left(c_{1}+c_{2}\right)-32\left(c_{1}^{2}+c_{1} c_{2}+c_{2}^{2}\right) .
$$

Remark 12. The controlled game that is constructed in this way can be interpreted as follows. Consider two firms who produce an identical product. Instead of selling the products themselves, the goods are sold on an instantaneously clearing market by a separate institution (the coordinator), who distributes the payoffs between the two firms using the decision rule $f$ (see Example 1).

From Eqs. (39)-(42) we find the HJBI-DAEs

$$
V_{1}^{\prime}=\frac{96-64 c_{1}-32 c_{2}}{\beta(1-\alpha)}
$$




$$
\begin{aligned}
& V_{2}^{\prime}=\frac{-96+32 c_{1}+64 c_{2}}{\beta \alpha}, \\
& V_{1}=\frac{\alpha}{r}\left(\left(c_{2}-c_{1}\right)\left(96-64 c_{1}-32 c_{2}\right)+\pi^{*}\left(c_{1}, c_{2}\right)\right), \\
& V_{2}=\frac{1-\alpha}{r}\left(\left(c_{1}-c_{2}\right)\left(96-32 c_{1}-64 c_{2}\right)+\pi^{*}\left(c_{1}, c_{2}\right)\right) .
\end{aligned}
$$

Similar HJBI-DAEs can be derived for the cases where one or more constraints on $c_{i}$ become active.

By starting the integration at $\alpha=\frac{1}{2}$ and using symmetry, the systems of HJBI-DAEs are solved using DASSL (see Brenan et al. 1989). In fact, we only calculate $V_{1}, V_{2}, c_{1}$ and $c_{2}$ for $\alpha$ from $\alpha=\frac{1}{2}$ to $\alpha=0.999$, (thus avoiding the singularity at $\alpha=1$ ), and then by using symmetry (i.e. $c_{1}(\alpha)=c_{2}(1-\alpha)$ ) we obtain the results for $\alpha=0.001$ to $\alpha=0.999$. The DASSL output is then fed into Matlab, where we use spline interpolation and the built-in Runge-Kutta ODE solver to simulate the resulting closed-loop dynamics of the controlled game.

We have already seen in the proof of Lemma 11 that in specifying consistent initial conditions the degree of freedom is 2 , i.e. when one of the pairs of variables $\left(V_{1}\left(\frac{1}{2}\right), V_{2}\left(\frac{1}{2}\right)\right)$ or equivalently $\left(c_{1}\left(\frac{1}{2}\right), c_{2}\left(\frac{1}{2}\right)\right)$ is chosen, the others are fixed by the system of HJBI-DAEs. By requiring the extra symmetry condition $c_{1}(\alpha)=c_{2}(1-\alpha), V_{1}(\alpha)=V_{2}(1-\alpha)$, i.e. $c_{1}\left(\frac{1}{2}\right)=c_{2}\left(\frac{1}{2}\right)$, the degree of freedom is reduced to 1 . In the experiments we have started by fixing the initial value of $c_{1}\left(\frac{1}{2}\right)=c_{2}\left(\frac{1}{2}\right)$, which then fully determines the consistent initial conditions.

In the experiments we have fixed the parameters $\beta=\frac{1}{3}$ and $r=1$. Note that by choosing $\beta>0$ the rule $f$ is such that the coordinator exhibits a kind of alluring behavior. If one of the players deviates more than the other, the coordinator will try to convince this player to cooperate more by offering him a new $\alpha$ which is more favorable to him.

We have varied the initial conditions $c_{1}\left(\frac{1}{2}\right)=c_{2}\left(\frac{1}{2}\right)$ (see Table 1). Consistent values $V_{1}\left(\frac{1}{2}\right)=V_{2}\left(\frac{1}{2}\right)$ are then calculated using the HJBI-DAEs Eqs. (53)-(56), evaluated in $\alpha=\frac{1}{2}$.

The results of the experiments are shown in Figs. 1-6. In Figs. 1 and 2 we see that for the initial conditions corresponding to $c_{1}\left(\frac{1}{2}\right)=c_{2}\left(\frac{1}{2}\right)=0.75$ and $c_{1}\left(\frac{1}{2}\right)=c_{2}\left(\frac{1}{2}\right)=0.79$, the solutions $V_{1}$ and $V_{2}$ become unbounded, and hence do not correspond to a stationary feedback Nash equilibrium of the controlled game. ${ }^{4}$ In the other cases (see Figs. 3-6), $V_{1}$ and $V_{2}$ are continuously differentiable and bounded, and hence correspond to stationary feedback Nash equilibria

\footnotetext{
${ }^{4}$ Note that $\pi^{*}$ is bounded and so the same holds for $\alpha \pi^{*}$ and $(1-\alpha) \pi^{*}$. Using the fact that the discount factor $r$ is positive, it immediately follows that any value function is necessarily bounded.
} 
Table 1

Some consistent initial conditions for $\beta=1 / 3, r=1$

\begin{tabular}{ll}
\hline$c_{1}\left(\frac{1}{2}\right)=c_{2}\left(\frac{1}{2}\right)$ & $V_{1}\left(\frac{1}{2}\right)=V_{2}\left(\frac{1}{2}\right)$ \\
\hline 0.75 & 45 \\
0.79 & 45.8832 \\
0.796 & 46.002432 \\
0.8 & 46.08 \\
0.9 & 47.52 \\
0.99 & 47.9952 \\
\hline
\end{tabular}

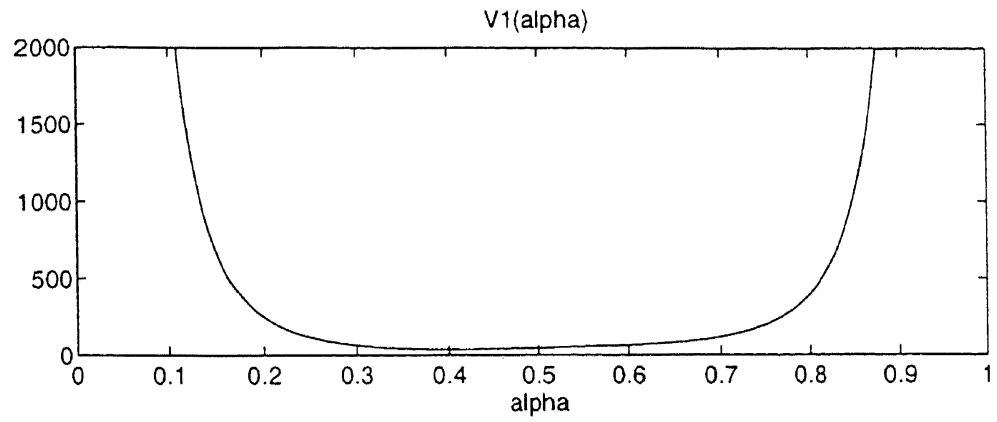

Optimal c

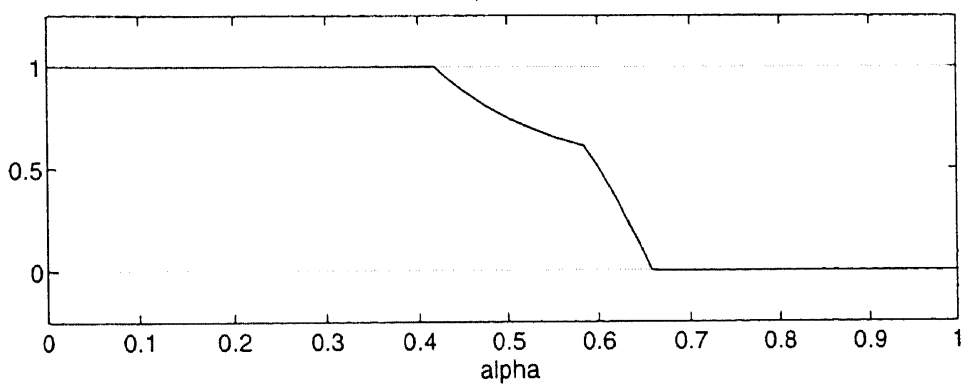

Fig. 1. $c_{1}\left(\frac{1}{2}\right)=c_{2}\left(\frac{1}{2}\right)=0.75$.

for the controlled game. In the Figs. 3-6 we have plotted $V_{1}(\alpha), c_{1}(\alpha)$, the closed-loop mechanism $f\left(\alpha, c_{1}(\alpha), c_{2}(\alpha)\right)$ and the simulated closed-loop dynamics $\dot{\alpha}(t)=f\left(\alpha(t), c_{1}(\alpha(t)), c_{2}(\alpha(t))\right)$ for $\alpha(0)=0.35,0.4,0.45,0.5,0.55,0.6$ and 0.65 .

In Figs. 3 and 4 we see that the corresponding stationary feedback Nash equilibria support five different steady states; three of these are unstable $(\alpha=0$, $\alpha=0.5$ and $\alpha=1)$, and two are stable $(\alpha \approx 0.4$ and $\alpha \approx 0.6)$. Finally, in Figs. 5 and 6 , the stationary feedback Nash equilibria support only three steady states; two of 


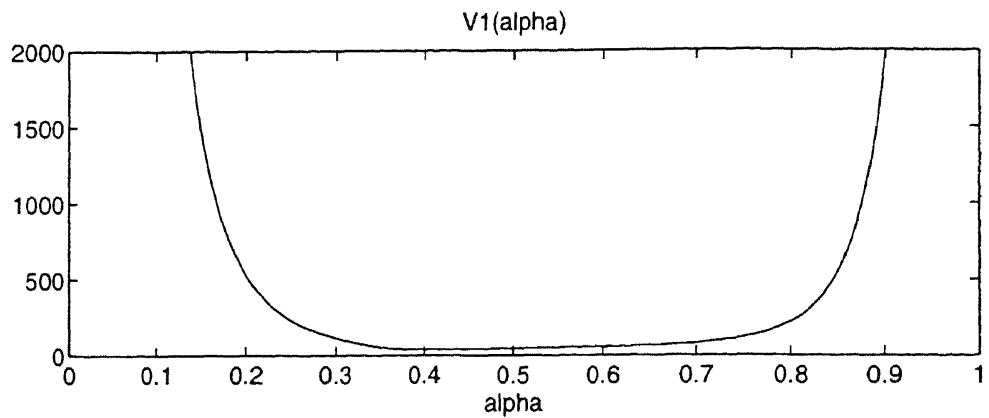

Optimal c

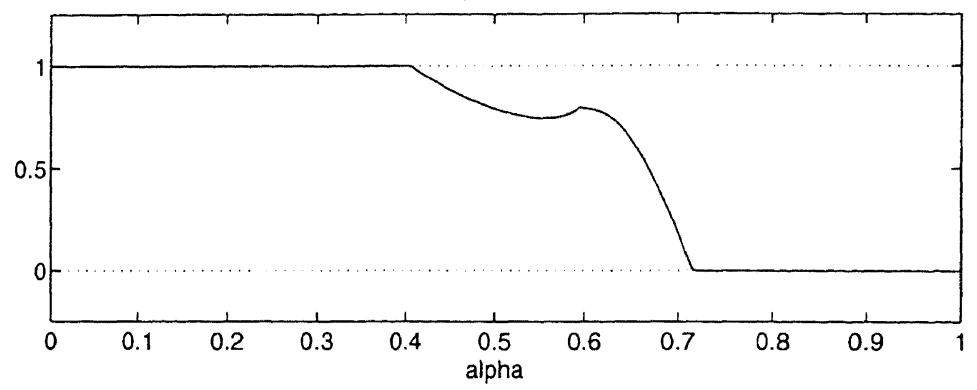

Fig. 2. $c_{1}\left(\frac{1}{2}\right)=c_{2}\left(\frac{1}{2}\right)=0.79$.
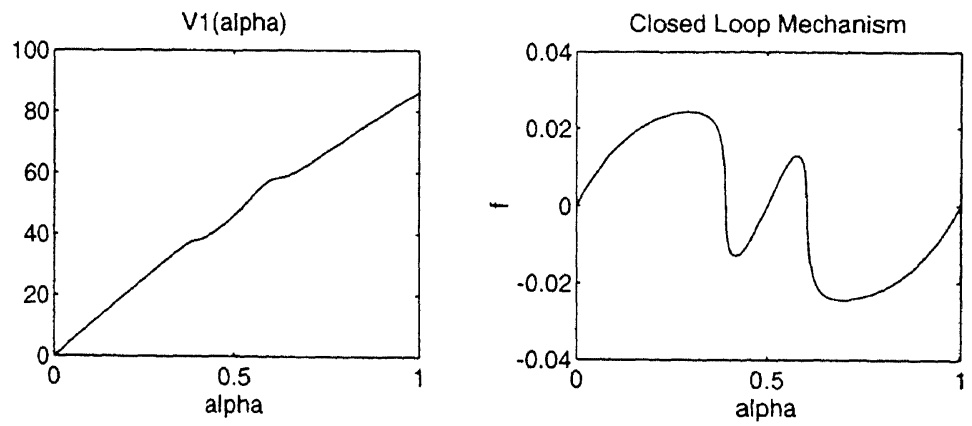

Optimal c1
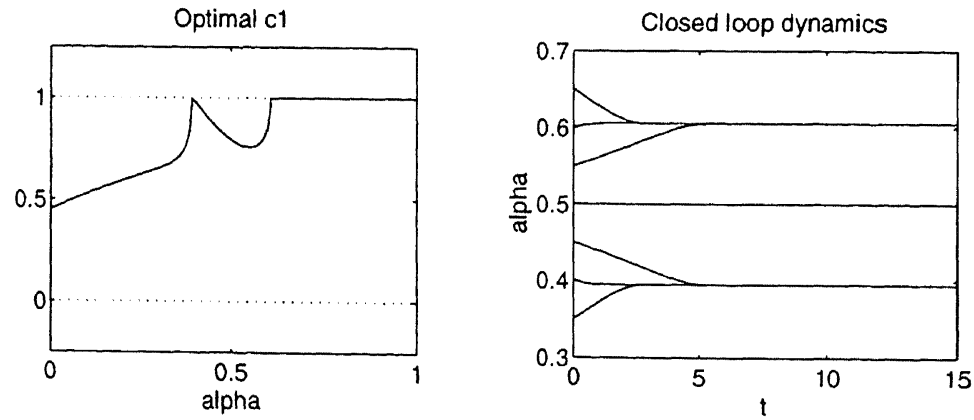

Fig. 3. $c_{1}\left(\frac{1}{2}\right)=c_{2}\left(\frac{1}{2}\right)=0.796$. 

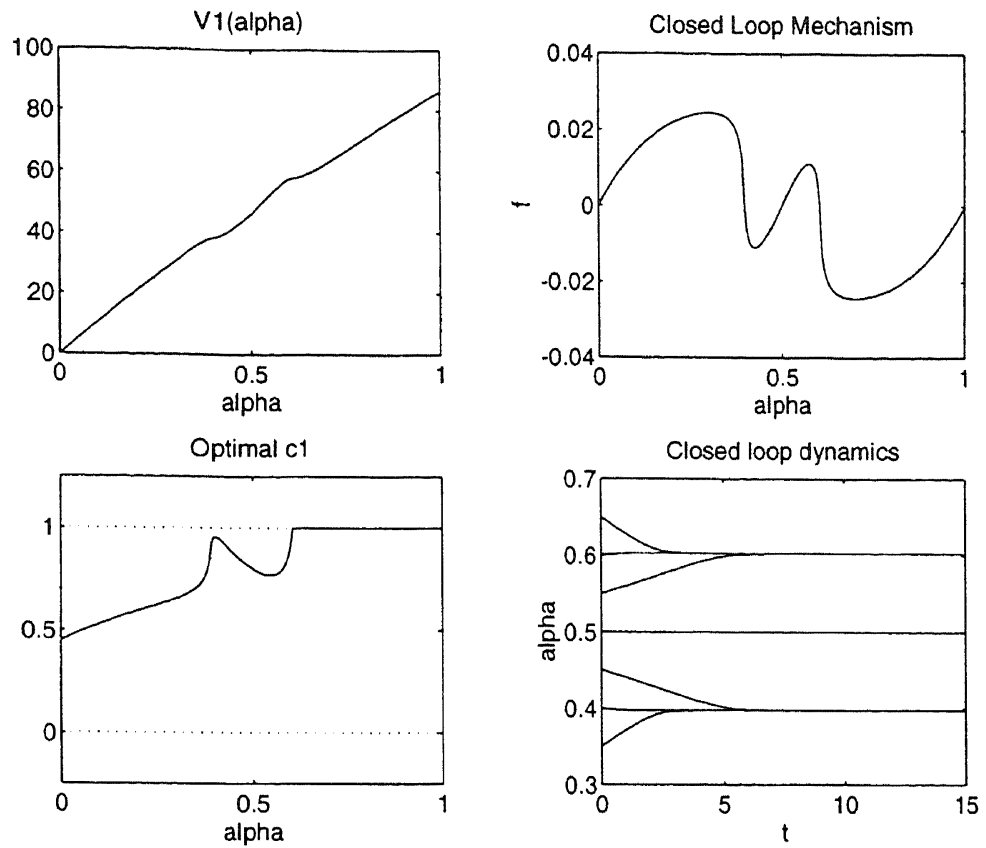

Fig. 4. $c_{1}\left(\frac{1}{2}\right)=c_{2}\left(\frac{1}{2}\right)=0.8$.
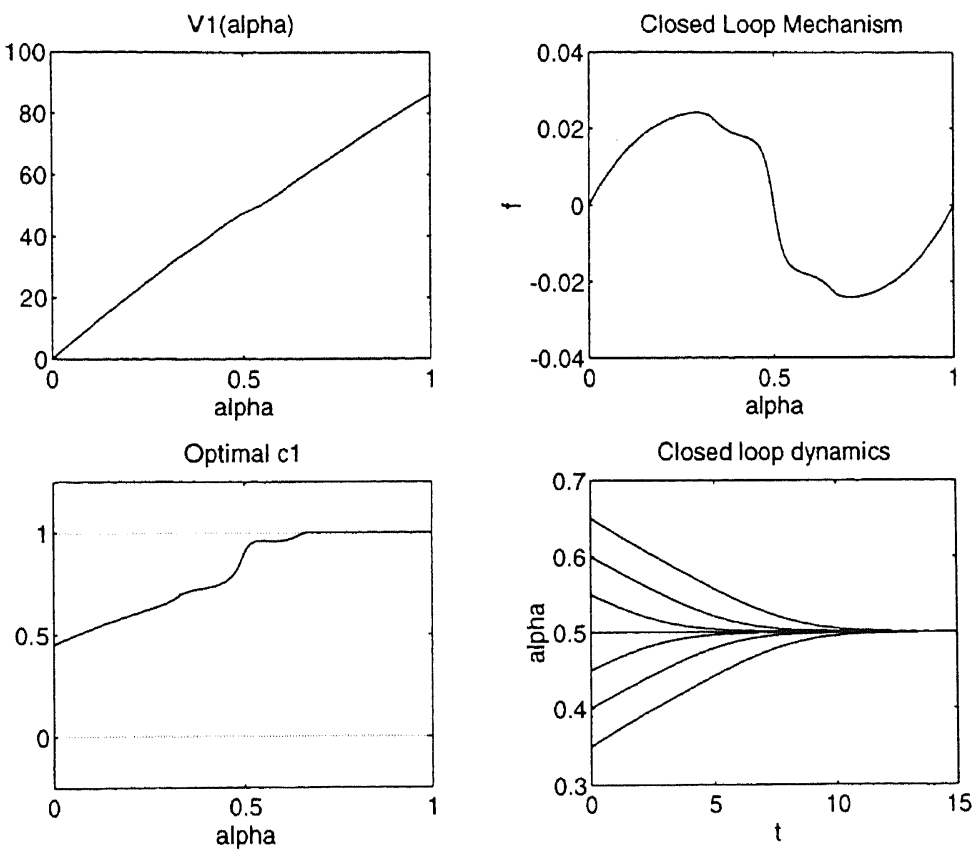

Fig. 5. $c_{1}\left(\frac{1}{2}\right)=c_{2}\left(\frac{1}{2}\right)=0.9$ 

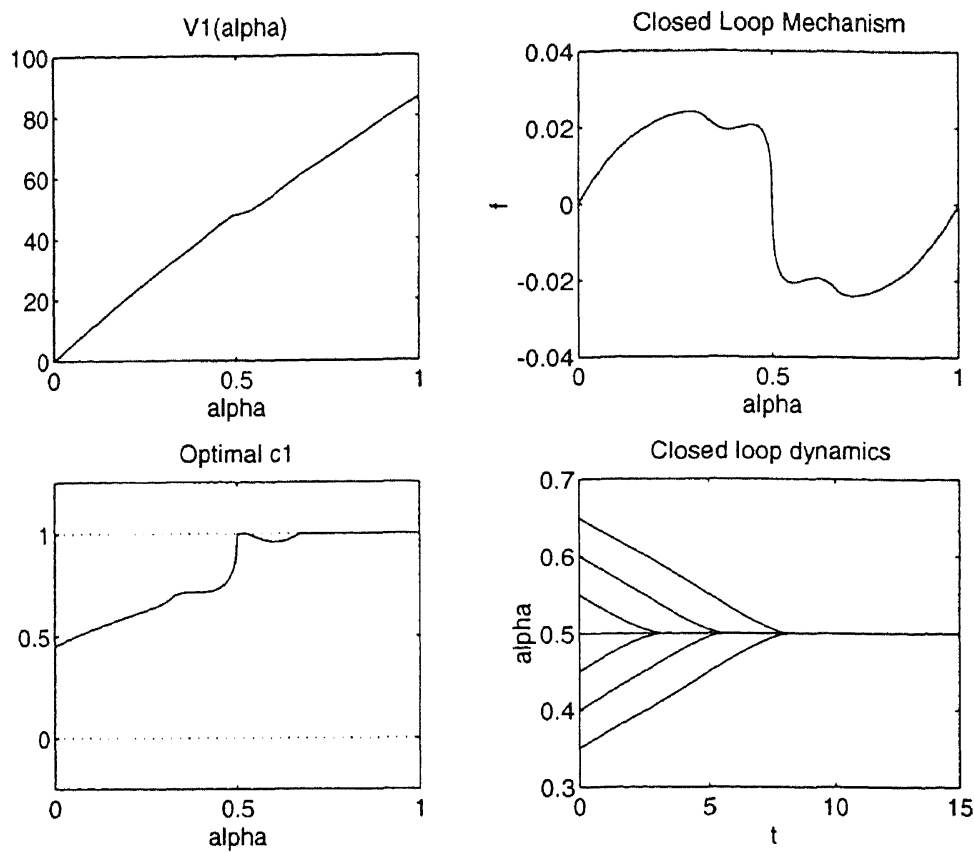

Fig. 6. $c_{1}\left(\frac{1}{2}\right)=c_{2}\left(\frac{1}{2}\right)=0.99$.

these are unstable $(\alpha=0$ and $\alpha=1)$ and one is stable $(\alpha=0.5)$. This suggests that somewhere between $c_{1}\left(\frac{1}{2}\right)=c_{2}\left(\frac{1}{2}\right)=0.9$ and $c_{1}\left(\frac{1}{2}\right)=c_{2}\left(\frac{1}{2}\right)=0.8$ a bifurcation takes place. To confirm this, we calculated the steady states belonging to equilibria corresponding to initial conditions $c_{1}\left(\frac{1}{2}\right)=c_{2}\left(\frac{1}{2}\right)$ ranging from 0.8 to 0.9. The outcomes are plotted in Fig. 7. In all the stable steady states we have also determined the corresponding values of the cooperation parameters $c_{1}=c_{2}$. These values are plotted in Fig. 8. In Fig. 9 we have plotted the payoffs for player 1 and 2 in the stable steady states which are greater than or equal to $\frac{1}{2}$. The dotted line in this figure is the payoff in the steady state $\frac{1}{2}$.

In all stationary feedback Nash equilibria that we find, convergence takes place to a quite cooperative situation; a threshold value of approximately 0.84 is found for the cooperation coefficients. However, there are two ways in which this cooperation is achieved. If the firms are already cooperative above the threshold value in a situation of equal distribution of profits $\left(\alpha=\frac{1}{2}\right)$, then a symmetric solution is obtained. This symmetry breaks down however if the firms are less cooperative at $\alpha=\frac{1}{2}$; the slightest deviation of the initial value $\alpha(0)=\frac{1}{2}$ will cause a process in which convergence takes place to a situation in which both firms are equally cooperative but take unequal shares in the revenues of cooperation. 


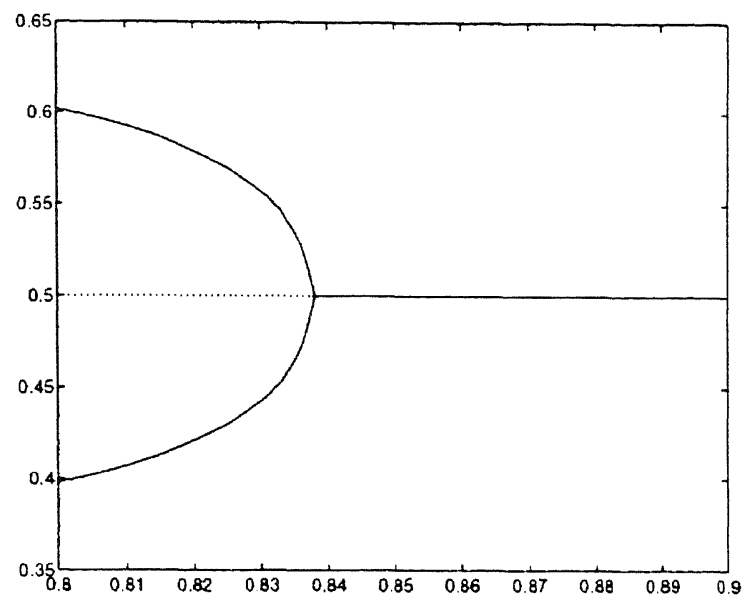

Fig. 7. Bifurcation of steady states.

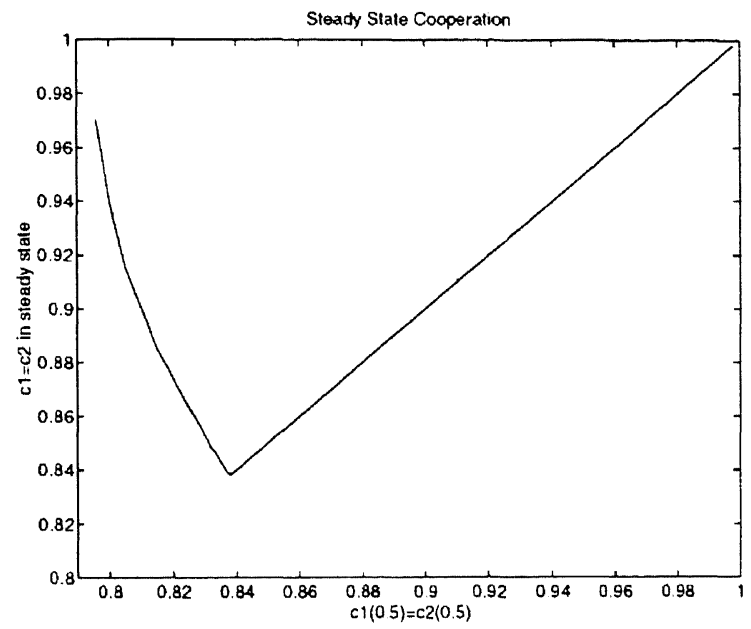

Fig. 8. Steady-state cooperation.

In a second experiment we have fixed the parameters at $r=1$ and $\beta=-\frac{1}{3}$. This results in a decision rule in which the coordinator punishes deviation. In this case it is easily verified that the strategies $c_{1}=c_{2} \equiv 1$ give a stationary feedback Nash equilibrium, with corresponding value functions $V_{1}(\alpha)=96 \alpha$ and $V_{2}(\alpha)=96(1-\alpha)$. Moreover, we calculated solutions of the HJBI equations corresponding to several initial conditions $c_{1}\left(\frac{1}{2}\right)=c_{2}\left(\frac{1}{2}\right) \in[0,1)$. In all these 

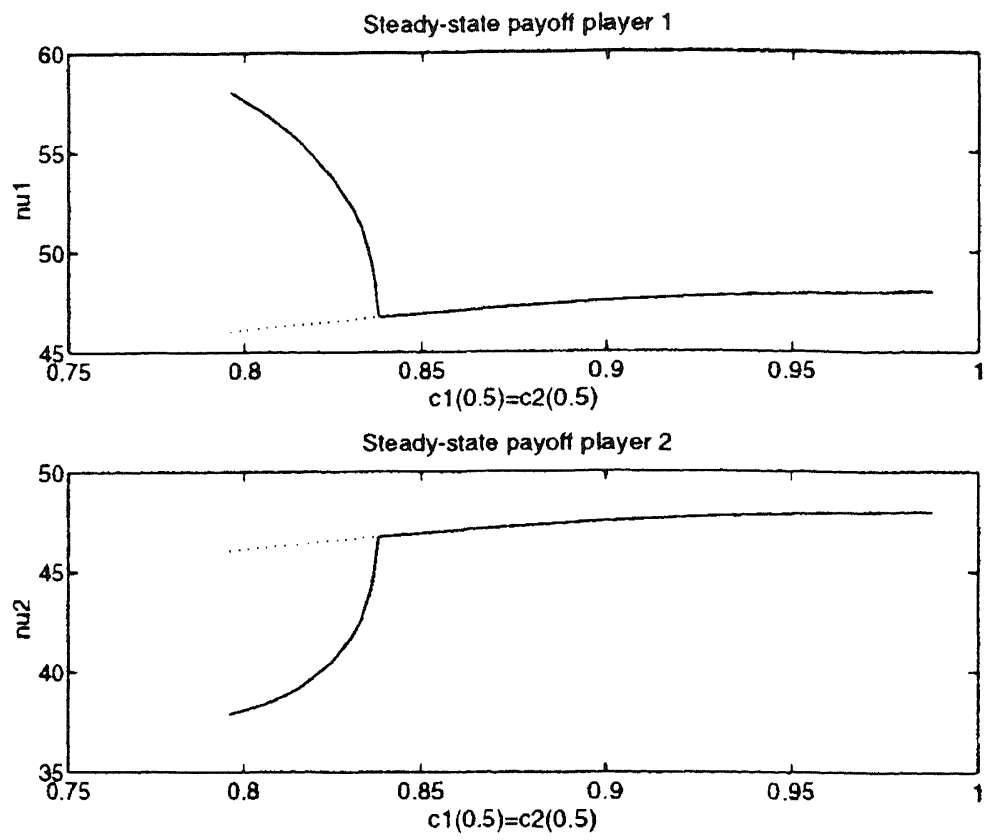

Fig. 9. Steady-state payoffs.

calculated solutions $V_{1}$ and $V_{2}$ turn out to be unbounded. This suggests that in this case the only symmetric stationary feedback Nash equilibrium is given by $c_{1}=c_{2} \equiv 1$. Apparently the mechanism in which the coordinator punishes any deviation from a joint cooperative strategy (i.e. the mechanism with $\beta<0$ ) is more effective, in the sense that it supports full cooperation (i.e. $c_{1}=c_{2} \equiv 1$ ) as the only symmetric stationary feedback Nash equilibrium of the controlled game.

\section{A Pareto mechanism}

In this section we will consider a situation in which the coordination parameter $\alpha$ affects the underlying static game $G$ not through the payoffs but rather through the strategy spaces of both players. We motivate the choice of coordination mechanism by the following result (see, e.g. Takayama 1985; Verkama 1994).

Theorem 13. For all $\mu \in(0,1)$ one has that if $\left(\hat{\gamma}_{1}, \hat{\gamma}_{2}\right) \in \Gamma_{1} \times \Gamma_{2}$ satisfies

$$
\left(\hat{\gamma}_{1}, \hat{\gamma}_{2}\right) \in \arg \max _{\left(\gamma_{1}, \gamma_{2}\right) \in \Gamma_{1} \times \Gamma_{2}}\left\{\mu \pi_{1}\left(\gamma_{1}, \gamma_{2}\right)+(1-\mu) \pi_{2}\left(\gamma_{1}, \gamma_{2}\right)\right\},
$$


then $\left(\hat{\gamma}_{1}, \hat{\gamma}_{2}\right)$ is Pareto efficient. Moreover, if $\Gamma_{1}, \Gamma_{2}$ are convex, and $\pi_{1}, \pi_{2}$ are concave, then for all Pareto efficient pairs $\left(\hat{\gamma}_{1}, \hat{\gamma}_{2}\right)$ there exists a $\mu \in[0,1]$ such that

$$
\left(\hat{\gamma}_{1}, \hat{\gamma}_{2}\right) \in \arg \max _{\left(\gamma_{1}, \gamma_{2}\right) \in \Gamma_{1} \times \Gamma_{2}}\left\{\mu \pi_{1}\left(\gamma_{1}, \gamma_{2}\right)+(1-\mu) \pi_{2}\left(\gamma_{1}, \gamma_{2}\right)\right\} .
$$

We will no longer assume that $G$ is symmetric. Also in this section we assume that the control objective is cooperation. The coordinator suggests which Pareto efficient strategy has to be chosen by the individual players, i.e. the coordinator determines the choice of $\mu$ according to Theorem 13 at time instant $t$. The cooperative strategies to be considered are $\gamma_{i}^{*}(\alpha)=\hat{\gamma}_{i}(\alpha)$. In this section we will exclude the possibility of sidepayments or redistribution, by taking

$$
v_{i}\left(\alpha, \gamma_{1}, \gamma_{2}\right)=\pi_{i}\left(\gamma_{1}, \gamma_{2}\right)
$$

The HJBI equations describing the stationary feedback Nash equilibria of the controlled game are given by

$$
\begin{aligned}
& r V_{1}(\alpha)=\max _{c_{1} \in[0,1]}\left\{V_{1}^{\prime}(\alpha) f\left(\alpha, c_{1}, c_{2}\right)+\pi_{1}\left(\gamma_{1}(\alpha), \gamma_{2}(\alpha)\right)\right\}, \\
& r V_{2}(\alpha)=\max _{c_{2} \in[0,1]}\left\{V_{2}^{\prime}(\alpha) f\left(\alpha, c_{1}, c_{2}\right)+\pi_{2}\left(\gamma_{1}(\alpha), \gamma_{2}(\alpha)\right)\right\} .
\end{aligned}
$$

We find the following proposition:

Proposition 14. Suppose $G$ has a unique Nash equilibrium $\left(\bar{\gamma}_{1}, \bar{\gamma}_{2}\right)$. Furthermore, suppose the alternative strategy $\gamma^{\mathrm{a}}$ is such that for all $\alpha \in(0,1)$ the system of equations

$$
\begin{aligned}
& c_{1}(\alpha) \gamma_{1}^{*}(\alpha)+\left(1-c_{1}(\alpha)\right) \gamma_{1}^{\mathrm{a}}(\alpha)=\bar{\gamma}_{1}, \\
& c_{2}(\alpha) \gamma_{2}^{*}(\alpha)+\left(1-c_{2}(\alpha)\right) \gamma_{2}^{\mathrm{a}}(\alpha)=\bar{\gamma}_{2},
\end{aligned}
$$

has a unique solution $\left(\bar{c}_{1}(\alpha), \bar{c}_{2}(\alpha)\right)$, with $0 \leq \bar{c}_{i}(\alpha) \leq 1$. Then a stationary feedback Nash equilibrium of the controlled game is given $b y$

$$
\left(c_{1}(\alpha), c_{2}(\alpha)\right)=\left(\bar{c}_{1}(\alpha), \bar{c}_{2}(\alpha)\right) .
$$

The actions $\left(\gamma_{1}(\alpha(t)), \gamma_{2}(\alpha(t))\right)$ played at every time instant $t$ are equal to the Nash equilibrium $\left(\bar{\gamma}_{1}, \bar{\gamma}_{2}\right)$ of $G$.

Proof. For all $\alpha \in(0,1)$ the Nash equilibrium of $G$ is recovered for $\left(c_{1}, c_{2}\right)=\left(\bar{c}_{1}(\alpha), \bar{c}_{2}(\alpha)\right)$, more precisely

$$
\begin{aligned}
& \bar{c}_{1}(\alpha) \in \underset{c_{1}}{\operatorname{argmax}} \pi_{i}\left(\gamma_{1}(\alpha), \bar{\gamma}_{2}(\alpha)\right), \\
& \bar{c}_{2}(\alpha) \in \underset{c_{2}}{\operatorname{argmax}} \pi_{i}\left(\bar{\gamma}_{1}(\alpha), \gamma_{2}(\alpha)\right) .
\end{aligned}
$$


Here $\bar{\gamma}_{i}(\alpha):=\bar{c}_{i}(\alpha) \gamma^{*}(\alpha)+\left(1-\bar{c}_{i}(\alpha)\right) \gamma^{,}(\alpha)$. Note that, for all $\alpha \in(0,1)$, $\pi_{i}\left(\bar{\gamma}_{1}(\alpha), \bar{\gamma}_{2}(\alpha)\right)=\pi_{i}\left(\bar{\gamma}_{1}, \bar{\gamma}_{2}\right)$ does not depend on $\alpha$. The HJBI equations are given by

$$
\begin{aligned}
& r V_{i}(\alpha)=\pi_{i}\left(\bar{\gamma}_{1}, \bar{\gamma}_{2}\right), \\
& V_{i}^{\prime}(\alpha)=0 .
\end{aligned}
$$

Remark 15. Note that although the actions at every time instant $t$ equal the actions corresponding to the unique Nash equilibrium of $G$, they emerge from a different strategy. Moreover, these equilibrium strategies can give rise to a nontrivial dynamic behavior of $\alpha$. So in this sense a coordination process does take place, but is never successful, because both players attain the same payoff as in the case of noncooperative play.

Remark 16. In general the conditions of Proposition 14 will not be satisfied for all $\alpha \in(0,1)$. In that case a stationary feedback Nash equilibrium of the controlled game will allow for different actions to be played. Also in the case of multiple Nash equilibria for the game $G$ different actions can be expected. Furthermore it is important to note that, even in the case that all the conditions of Proposition 14 are fulfilled, this Nash equilibrium is not necessarily unique.

In the specific Cournot duopoly example we used in the previous section, it can straightforwardly be shown that the stationary feedback Nash equilibrium described by Proposition 14, in case the alternative strategies $\gamma_{i}^{\mathrm{a}}$ are chosen to be equal to the Nash strategies $\bar{\gamma}_{i}$, is in fact unique. This is a consequence of the fact that $c_{1}\left(\frac{1}{2}\right)=c_{2}\left(\frac{1}{2}\right)=0$ provides the only consistent initial condition for the HJBI-DAEs. In this case the determinant of the matrix $\mathscr{J}$ (see Eq. (29)) is given by

$$
\operatorname{det} \mathscr{J}=-331776 \frac{16 \alpha^{4}-32 \alpha^{3}+3 \alpha^{2}+13 \alpha-4}{\left(1-16 \alpha+16 \alpha^{2}\right)^{2}}
$$

so that $\operatorname{det} \mathscr{J}=0$ for $\alpha=-\frac{1}{8}+\frac{1}{8} \sqrt{17} \approx 0.390$ or for $\alpha=\frac{9}{8}-\frac{1}{8} \sqrt{17} \approx 0.610$, and moreover the denominator of $\operatorname{det} \mathscr{J}$ equals 0 for $\alpha=\frac{1}{2} \pm \frac{1}{4} \sqrt{3}$. Hence the system of HJBI-DAEs is locally of higher index. As a consequence the DASSL code cannot be used to obtain the solution. However, using the code RADAU5 (see Hairer et al. 1989; Hairer and Wanner, 1991), which is also suited for semi-explicit index 2 and index 3 systems, it is possible to find the solution efficiently. ${ }^{5}$

\footnotetext{
${ }^{5}$ Of course in this case we did not need to use any numerical method to find the solution. However, we can use this case as a testcase for the different numerical methods for DAEs.
} 


\section{Conclusions}

In this paper we have considered a class of models describing noncooperative hierarchical control. The most important aspect in this setup is that we have allowed for strategic behavior by the individual players, influencing the outcome of the coordination process. In the context of continuously repeated games we have obtained a nonlinear differential game, with state variable $\alpha$, which we called the controlled game.

We have taken a closer look at two special cases of such a controlled game, namely a redistribution controlled game and a Pareto controlled game. In both these special cases we looked at the control objective of establishing cooperation between the players in order to maximize efficiency. Using recently developed methods for differential-algebraic equations (DAEs), we have described in what way all stationary feedback Nash equilibria can be calculated for such differential games. In a worked example of a repeated symmetric Cournot duopoly, we have illustrated the numerical method for the redistribution controlled game. We saw that for this example there are several qualitatively different stationary feedback Nash equilibria. In this example we saw that if the players are sufficiently willing to cooperate at the point where all extra payoffs are divided equally between the players, this point is supported by the stationary feedback Nash equilibria as the only stable steady state. However, in case the players are not sufficiently willing to cooperate at this point, the stability of the steady state is lost. Moreover, we have seen that if the coordinator's decision rule is changed in such a way that relatively noncooperative attitudes are punished by the coordinator, a symmetric stationary feedback Nash equilibrium exists that supports full cooperation of both players. In the case of the Pareto controlled game, we found for the same example that the unique stationary feedback Nash equilibrium does not support any cooperation at all; in this case the coordination mechanism is too weak to stimulate cooperation. We can conclude that the choice of coordination mechanism and the choice of decision rule for the coordinator can be viewed as a control problem; by choosing the appropriate mechanism and decision rule a global control objective can be pursued.

The models as introduced in this paper are capable of describing hierarchical situations where a coordinator is using a given (fixed) decision rule in order to reach some given control objective. In the context of continuously repeated games, we have shown how such a hierarchical situation can be translated into a differential game. For these games we have introduced a new method to calculate the stationary feedback Nash equilibria. The behavior of the corresponding variables of interest will depend on the strategy chosen by the coordinator, and in this way one obtains a method for policy evaluation.

Obviously the model used in this paper is relatively simple and it would be natural to think of several extensions. In all examples we have looked at situations with only two players; the introduction of more than two players is 
fairly straightforward as long as coalitions are not allowed. When coalitions may be formed, however, the analysis becomes much more difficult. The introduction of more players might also lead to a state variable with more than one component; for instance this would be natural if the state is somehow interpreted as an indication of the position of the coordinator between the players. In this case the basic problem may still be posed as in the present paper but its numerical complexity would be much higher, since the HJBI equations lead in that case to partial differential equations rather than to ordinary differential equations. As a possible specialization of the models used in this paper, one may mention the case in which a zero-sum game is played on the lower decision level; this could be used for instance as a model for a Stackelberg game with a risk-sensitive follower.

An important limitation of our models is that they are deterministic. In practice, a coordinator often tries to prevent strategic behavior by leaving some uncertainty about his responses to actions by the players. It would be of interest to investigate theoretically the effectivity of such a use of uncertainty.

\section{References}

Başar, T., Olsder, G.J., 1995. Dynamic Noncooperative Game Theory, 2nd ed. Academic Press, London.

Bagchi, A., 1984. Stackelberg Differential Games in Economic Models. Lecture Notes in Control and Information Science, vol. 64. Springer, Berlin.

Brasey, V, Hairer, E, 1993. Half-explicit Runge-Kutta methods for differential-algebraic systems of index 2. SIAM Journal of Numerical Analysis 30, 538-552.

Brenan, K.E, Campbell, S.L, Petzold, L.R, 1989. Numerical Solution of Initial-Value Problems in Differential-Algebraic Equations. North-Holland, New York.

Feichtinger, G, Wirl, F, 1993. A dynamic variant of the battle of the sexes. International Journal of Game Theory 22, 359-380.

Gear, C.W, 1988. Differential-algebraic index transformations. SIAM Journal of Scientific and Statistical Computations 9, 39-47.

Gibbons, R, 1992. A Primer in Game Theory. Harvester Wheatsheaf, New York.

Griepentrog, E., März, R., 1986. Differential-Algebraic Equations and Their Numerical Treatment. Teubner-Texte zur Mathematik, vol. 88. Teubner, Leipzig.

Hairer, E., Lubich, C., Roche, M., 1989. The Numerical Solution of Differential-Algebraic Systems by Runge-Kutta Methods. Lecture Notes in Mathematics, vol. 1409. Springer, Berlin.

Hairer, E., Wanner, G., 1991. Solving Ordinary Differential Equations II, Stiff and DifferentialAlgebraic Problems. Springer Series in Computational Mathematics, vol. 14. Springer, Berlin.

Ho, Y.-C, Luh, P, Olsder, G, 1982. A control-theoretic view on incentives. Automatica 18 , $167-179$.

Houba, H., 1994. Game Theoretic Models of Bargaining. Ph.D. Thesis, Tilburg University.

Jamshidi, M., 1983. Large-Scale Systems, Modeling and Control. System Science and Engineering. vol. 9. North-Holland, New York.

Lucas, R.E., 1976. Econometric policy evaluation: a critique. In: Brunner, K., Meltzer, A.M., (Eds.), The Phillips Curve and Labor Markets. Carnegie-Rochester Conferences on Public Policy, vol. 1. North-Holland, Amsterdam, pp. 19-46. 
Maskin, E., Tirole, J., 1994. Markov perfect equilibrium. In: Breton, M., Zaccour, G., (Eds.), Preprint Volume of the 6th International Symposium on Dynamic Games and Applications, pp. 432-461.

Mesarovic, M.D, Macko, D, Takahara, Y, 1970. Theory of Hierarchical Multilevel Systems. Academic Press, New York.

Osborne, M, Rubinstein, A, 1991. Bargaining and Markets. Academic Press, Boston.

Singh, M.G, 1980. Dynamical Hierarchical Control. North-Holland, Amsterdam.

Takayama, A., 1985. Mathematical Economics, 2nd ed. Cambridge University Press, Cambridge.

Tsutsui, S, Mino, K, 1990. Nonlinear strategies in dynamic duopolistic competition with sticky prices. Journal of Economic Theory 52, 136-161.

van Damme, E., 1991. Stability and Perfection of Nash Equilibria, 2nd ed. Springer, Berlin.

Verkama, M., 1994. Distributed methods and processes in games of incomplete information. Ph.D. Thesis, Helsinki University of Technology.

Weeren, A.J.T.M., Schumacher, J.M., Engwerda, J.C., 1994. Asymptotic analysis of Nash equilibria in nonzero-sum linear-quadratic differential games. The two-player case. Research Memorandum FEW 634, Tilburg University. 